Article

\title{
Application of Solution Plasma Surface Modification Technology to the Formation of Thin Hydroxyapatite Film on Titanium Implants
}

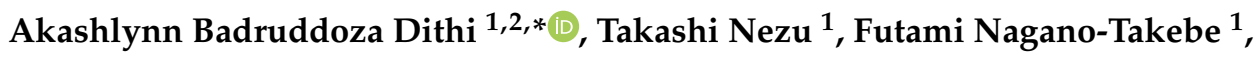 \\ Md Riasat Hasan ${ }^{2}\left(\mathbb{D}\right.$, Takashi Saito ${ }^{2}$ (D) and Kazuhiko Endo ${ }^{1}$ \\ 1 Division of Biomaterials and Bioengineering, Department of Oral Rehabilitation, School of Dentistry, \\ Health Sciences University of Hokkaido, 061-0293 Hokkaido, Japan; tnezu@hoku-iryo-u.ac.jp (T.N.); \\ nagano23@hoku-iryo-u.ac.jp (F.N.-T.); endo@hoku-iryo-u.ac.jp (K.E.) \\ 2 Division of Clinical Cariology and Endodontology, Department of Oral Rehabilitation, School of Dentistry, \\ Health Sciences University of Hokkaido, 061-0293 Hokkaido, Japan; riasat@hoku-iryo-u.ac.jp (M.R.H.); \\ t-saito@hoku-iryo-u.ac.jp (T.S.) \\ * Correspondence: akashlynn@hoku-iryo-u.ac.jp; Tel./Fax: +81-0133-23-1726
}

Received: 21 September 2018; Accepted: 13 December 2018; Published: 21 December 2018

\begin{abstract}
Hydroxyapatite (HA) coatings on titanium implants enhance rapid bone formation around the implant due to their osteoconductive property. The present study aimed to achieve a thin and uniform HA film coating on titanium implants by solution plasma treatment (SPT). Commercially pure titanium and porous titanium disks were employed. A pulse plasma generator was used on the disks for $30 \mathrm{~min}$. Morphologic and crystallographic features of the deposited films were examined by scanning electron microscopy (SEM) and X-ray diffractometry (XRD). To evaluate the wettability of the disks, water droplet $(20 \mu \mathrm{L})$ surfaces were measured using a contact angle analyzer. The initial attachment of osteoblast-like cells (MC3T3E1) on the titanium substrates before and after solution plasma treatment was evaluated by counting the number of attached cells after incubation for $4 \mathrm{~h}$. After immersion in the mineralizing solution for up to seven days, no crystals were observed on the polished-Ti surface. A more uniform and dense precipitation of round and grown crystals with diameters of approximately $1-5 \mu \mathrm{m}$ was observed on Ti-SPT. XRD clearly showed that the precipitated crystals on titanium disks were HA. The contact angle of the polished-Ti increased with time $\left(\theta=37^{\circ}-51^{\circ}\right)$. The surface of the Ti-SPT remained hydrophilic $\left(\theta<5^{\circ}\right)$ after up to 30 days of aging. The number of attached cells on the Ti-SPT after aging for 30 days remained above $85 \%$ of that on the Ti-SPT without aging. SPT in a mineralizing solution can be used to acquire a homogenous precipitation of HA on porous-surfaced titanium implants.
\end{abstract}

Keywords: hydroxyapatite; titanium implants; mineralizing solution; solution plasma treatment

\section{Introduction}

The use of dental implants has revolutionized the current treatment of partially and fully edentulous patients given their high level of predictability and wide variety of treatment options. In 2006, the estimated number of dental titanium implants placed in the United States was over five million [1]. A major consideration in designing dental implants is the production of surfaces that promote desirable responses in the cells and tissues.

Hydroxyapatite (HA) coatings on titanium implants facilitate rapid bone formation due to their excellent osteoconductive property. HA-coated implants have been reported to stimulate bone healing, which enhances improvement in the rate and strength of the initial implant integration. For enhanced implant stability and bio-integration to bone tissue, various methods have been 
applied to coat the titanium implant with HA, for example, plasma spraying [2], electrophoretic co-deposition [3], ion-beam-sputter deposition [4], dip coating in a simulated body fluid [5], electrochemical deposition [6], blast coating [7], and thermally induced liquid-phase deposition [8,9]. Among these methods, titanium implant bodies coated with HA in dry processes, such as the plasma spraying and ion-beam-sputter deposition methods, have already been used clinically. The HA coating methods in dry processes, however, cannot be applied to titanium implant bodies with porous surface structures. To coat titanium implants with a three-dimensional porous surface structure with HA film, Kuroda et al. and Tamura et al. developed the titanium substrate heating method in a liquid $[8,9]$. Since the solubility product of HA decreases with an increase in temperature above $16^{\circ} \mathrm{C}$, they electrically heated the titanium substrate by applying a large current with an AC or DC power source to the substrate in calcium phosphate solutions to precipitate HA crystals on the titanium surface. These two studies demonstrated that the precipitated HA crystals were needle-like or plate-like in shape and that the formation of a uniform and dense HA film consisting of fine and spherical HA crystals could not be achieved. Another shortcoming of the titanium substrate heating method is that it requires a large electric power source exceeding $1000 \mathrm{~V}$ for the deposition of HA crystals on the surface of the dental implant body by Joule heating.

The solution plasma technique is an entirely new technology to coat an implant surface with HA spherical particles. To date, this solution plasma technique has been used in the fields of chemistry and applied physics [10]. When a pulsed voltage is applied between the two electrodes, a glowing discharge takes place in the liquid, which generates plasma in the gas phase. This plasma produces thermal energy and light, along with reactive chemical species such as hydrogen and hydroxide $(\mathrm{OH})$ ions. With this novel surface modification technique, the temperature of the solution in contact with the titanium surface can easily be increased with the thermal energy generated by the solution plasma. In this case, a thin and uniform HA layer is expected to be formed on a porous-surfaced titanium implant, which will induce rapid bone growth into the pore space of the implant body, and also enhance the biological anchorage of the implant with bone.

The present study aimed to achieve a thin and uniform HA layer covering titanium with a porous surface structure by using solution plasma technology. The mechanism of HA precipitation on the titanium during solution plasma treatment was elucidated.

\section{Materials and Methods}

\subsection{Substrate}

Commercially pure titanium and porous titanium with $69.7 \%$ porosity were employed in this study.

Group A: Non-porous-Ti (Ti) (JIS Type-2, J. Morita Corp., Tokyo, Japan); spherical shape; specimen size was $14 \mathrm{~mm}$ in diameter and $2 \mathrm{~mm}$ in thickness.

Group B: Porous-Ti (powder sintering product with 69.7\% porosity; Nagamine Manufacturing Company, Kagawa, Japan); specimen size was $14 \mathrm{~mm}^{2}, 1 \mathrm{~mm}$ in thickness, and the pore size was $287 \pm 87 \mu \mathrm{m}^{2}$.

The Ti disks were polished with silicon carbide abrasive papers (\#240, then \#600). The polished groups were named "Polished-Ti". "Porous-Ti" was used without polishing for the experiments.

Half of the polished-Ti and porous-Ti substrates were subjected to alkaline treatment prior to the solution plasma treatment (SPT). The disks were immersed in $5 \mathrm{M} \mathrm{NaOH}$ solution at $60{ }^{\circ} \mathrm{C}$ for $24 \mathrm{~h} \mathrm{[11].} \mathrm{The} \mathrm{alkaline} \mathrm{treatment} \mathrm{formed} \mathrm{a} \mathrm{micrometric} \mathrm{network} \mathrm{layer} \mathrm{of} \mathrm{sodium} \mathrm{titanate} \mathrm{hydrogel} \mathrm{on}$ the titanium surface, which enhanced the apetite nucleation in mineralizing solution.

After alkaline treatment, the polished titanium (Ti) group was named "Ti-AT" and the porous titanium substrate (porous-Ti) group was named "Porous-Ti-AT". 


\subsection{Preparation of the Mineralizing Solution}

A metastable calcium phosphate solution was used in this study [12]. The composition of the solution is shown in Table 1 . The $\mathrm{Ca} / \mathrm{P}$ molar ratio of the mineralizing solution was 1.67 , and it also contained $10 \mathrm{mM}$ HEPES ( $N$-2-Hydroxyethylpiperazine- $N^{\prime}$-2-Ethanesulfonic acid) $\mathrm{KOH}$ (potassium hydroxide) for buffering at $\mathrm{pH}$ 7.4. The final ionic strength of this solution was adjusted to 0.16 by adding $150 \mathrm{mM} \mathrm{KCl}$ (potassium chloride). The preparation of the solution was completed by dissolving reagent grade $\mathrm{CaCl}_{2}$ (calcium chloride), $\mathrm{KH}_{2} \mathrm{PO}_{4}$ (potassium phosphate), $\mathrm{KCl}$ (potassium chloride), and HEPES (N-2-Hydroxyethylpiperazine- $N^{\prime}$-2-Ethanesulfonic acid) into deionized distilled water. The degree of supersaturation with respect to hydroxyapatite was $3.85 \times 10^{7}$ at $37^{\circ} \mathrm{C}$.

Table 1. Composition of the mineralizing solution.

\begin{tabular}{cc}
\hline Component & Concentration (mM) \\
\hline $\mathrm{CaCl}_{2}$ & 2.24 \\
$\mathrm{~K}_{2} \mathrm{HPO}_{4}$ & 1.04 \\
$\mathrm{KH}_{2} \mathrm{PO}_{4}$ & 0.30 \\
$\mathrm{HEPES}$ & 10.00 \\
$\mathrm{KCl}$ & 150.00 \\
$\mathrm{C} / \mathrm{P}$ & 1.67 \\
$\mathrm{pH}$ & 7.40 \\
Degree of supersaturation with respect to HA & $3.85 \times 10^{7}$ \\
\hline
\end{tabular}

\subsection{Solution Plasma Treatment}

A solution plasma treatment was performed in $150 \mathrm{~mL}$ of the mineralizing solution at $29^{\circ} \mathrm{C}$. A pulse plasma generator (MPP-NV04, Pekuris, Tokyo, Japan) was used and the polished-Ti and porous-Ti substrates were placed at a distance of $5 \mathrm{~mm}$ from the electrodes within a glass vial (Figure 1). Plasma was generated at a voltage of $5 \mathrm{~V}$ and pulse width of $3 \mu \mathrm{s}$ for $30 \mathrm{~min}$. The mineralizing solution was stirred using a magnetic stirrer (Ion Stir 7d, Central Kagaku Co., Ltd., Tokyo, Japan) at a rotational speed of $240 \mathrm{rpm}$. The duration of the solution plasma treatment was $30 \mathrm{~min}$.

When the solution plasma surface modification technique was applied to "Ti-AT" and on "Porous-Ti-AT", they were named "Ti-AT-SPT" and "Porous-Ti-AT-SPT", respectively.

(a)
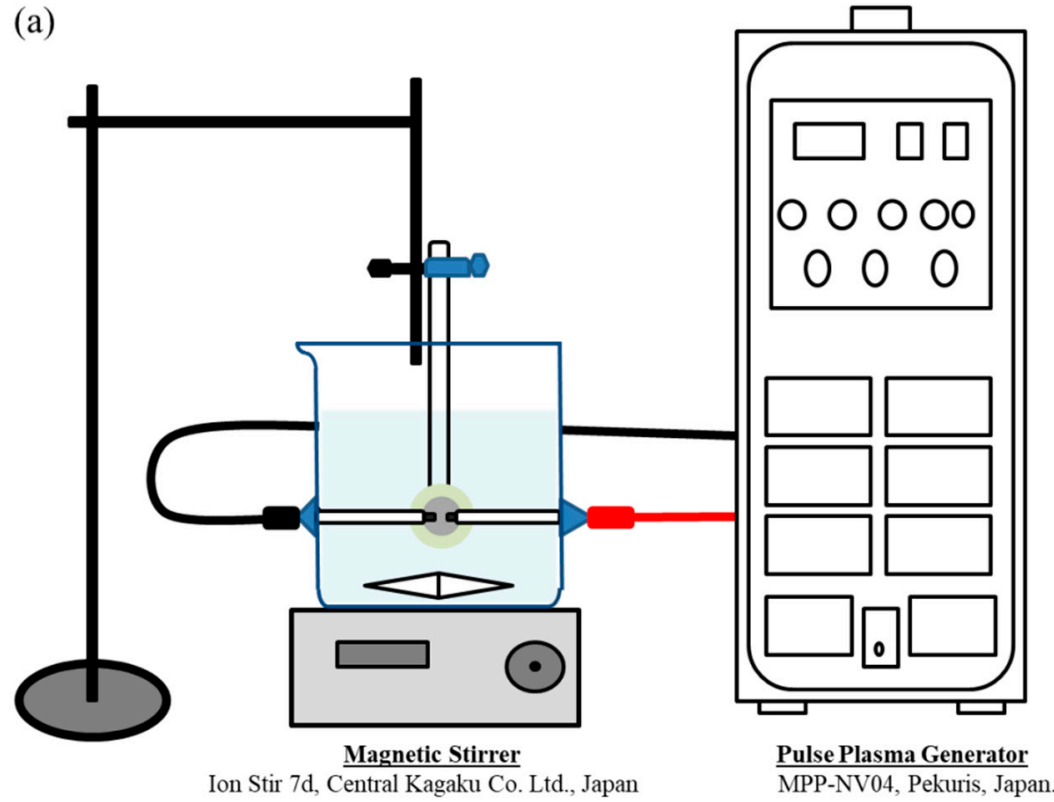

Figure 1. Cont. 
(b)

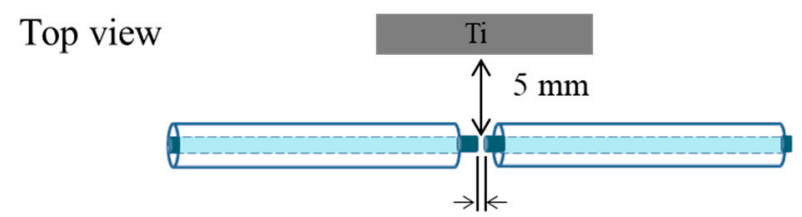

(c)

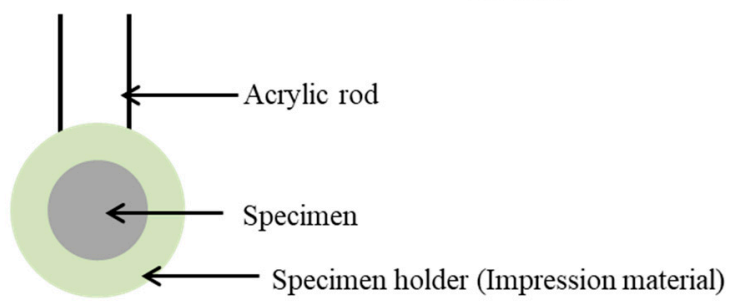

Figure 1. Apparatus, electrodes, and specimen employed for the solution plasma treatment. The specimen was placed at a 5 -mm distance from the electrodes in a glass vial containing $150 \mathrm{~mL}$ of the mineralizing solution. Plasma was generated at $5 \mathrm{~V}$ and $3 \mu \mathrm{m}$ pulse width for $30 \mathrm{~min}$. (a) Pulse plasma generator, Magnetic stirrer, Holder stand; (b) Electrodes (2 electrode having $0.5 \mathrm{~mm}$ distance between them; (c) The specimen holder is made of Duplicone and acrylic rods help to hold the specimen holder.

\subsection{Characterization of the HA Layer Formed on Various Substrates}

The morphologic and crystallographic features of deposited films were examined by SEM and XRD. The contact angle of the water droplets $(20 \mu \mathrm{L})$ on the titanium surfaces was measured by a contact angle analyzer after each surface treatment.

\subsubsection{SEM Observation}

The morphology of the titanium surface after SPT and the immersion treatments was examined using a scanning electron microscope (SSX-550, Shimadzu Corporation, Kyoto, Japan) with an acceleration voltage of $10 \mathrm{kV}$, after the samples were coated with gold.

\subsubsection{X-ray Diffraction}

The crystallographic features of the deposited films were examined using an X-ray diffractometer (JDX-3500, JEOL Ltd., Tokyo, Japan) with $\mathrm{Cu} K \alpha$ radiation $(\lambda=0.1541 \mathrm{~nm})$ at room temperature. The $2 \theta$ range was $20-80^{\circ}$, and the XRD profile was recorded at step-scan intervals of $0.02^{\circ}$ at a scanning speed of $8.0^{\circ} / \mathrm{min}$ at $40 \mathrm{kV}$ and $150 \mathrm{~mA}$.

\subsubsection{Measurement of the Contact Angle of Water Droplets on the Surfaces}

To evaluate the wettability of the titanium disks before and after surface treatment, the contact angle of the water droplets $(20 \mu \mathrm{L})$ on the surfaces was measured using a contact angle analyzer (Phoenix Alpha, Seoul, Korea) at 0, 3, 7, and 14 days after each surface treatment.

\subsection{Evaluation of Cytocompatibility}

\subsubsection{Cell Line}

The MC3T3E1 cell line was used in this study to evaluate the cell attachment of the titanium samples before and after SPT.

2.5.2. Attachment of Cells on Differently Modified Titanium Surfaces after Four Hours of Incubation

There were 6 groups for each experiment, that is, 30-day aged ("Polished-Ti" and "Ti-AT-SPT"), 7-day aged ("Polished-Ti" and "Ti-AT-SPT"), fresh "Polished-Ti", and fresh "Ti-AT-SPT". Four specimens from each group were placed on a 24 -well plate, disinfected with $1 \mathrm{~mL}$ of $70 \%$ ethanol 
for $10 \mathrm{~min}$ and washed with sterilized distilled water for $10 \mathrm{~min}$. The number of MC3T3E1 cells was adjusted to be $5 \times 10^{4}$ cells $/ \mathrm{mL}$ in a regular medium consisting of $\alpha$-modified minimum essential medium ( $\alpha$-MEM; with L-glutamine and phenol red, Wako Pure Chemical Industries Ltd., Osaka, Japan) supplemented with 10\% heat-inactivated fetal bovine serum. Adjusted cells were seeded on a sample with $1 \mathrm{~mL}$ per well. After $4 \mathrm{~h}$ of incubation under a humidified atmosphere of $5 \% \mathrm{CO}_{2}$ at $37^{\circ} \mathrm{C}$, the cells were detached by trypsin-EDTA (0.25\% Trypsin/0.53 mM EDTA in Hanks Balanced Salt Solution without calcium or magnesium) and the number of cells was counted by a hemocytometer.

\subsubsection{Statistical Analysis}

Statistical analysis was performed using one-way analysis of variance (ANOVA), and the Games-Howell post hoc test with the significance level of $p<0.05$.

\section{Results}

\subsection{Variation of Solution Temperature in the Vicinity of the Sample with Time during SPT}

Figure 2 shows the variation of the solution temperature with time during SPT measured by a thermocouple placed in the vicinity of the sample. The temperature increased rapidly and exceeded 60 ${ }^{\circ} \mathrm{C}$ within 3 min after starting SPT, and then increased slowly and reached an almost constant value of $80{ }^{\circ} \mathrm{C}$ at $30 \mathrm{~min}$. After $30 \mathrm{~min}$ of SPT, crystals were precipitated due to heterogeneous nucleation both on the sample surface and in the solution.

\section{Temparature change during SPT}

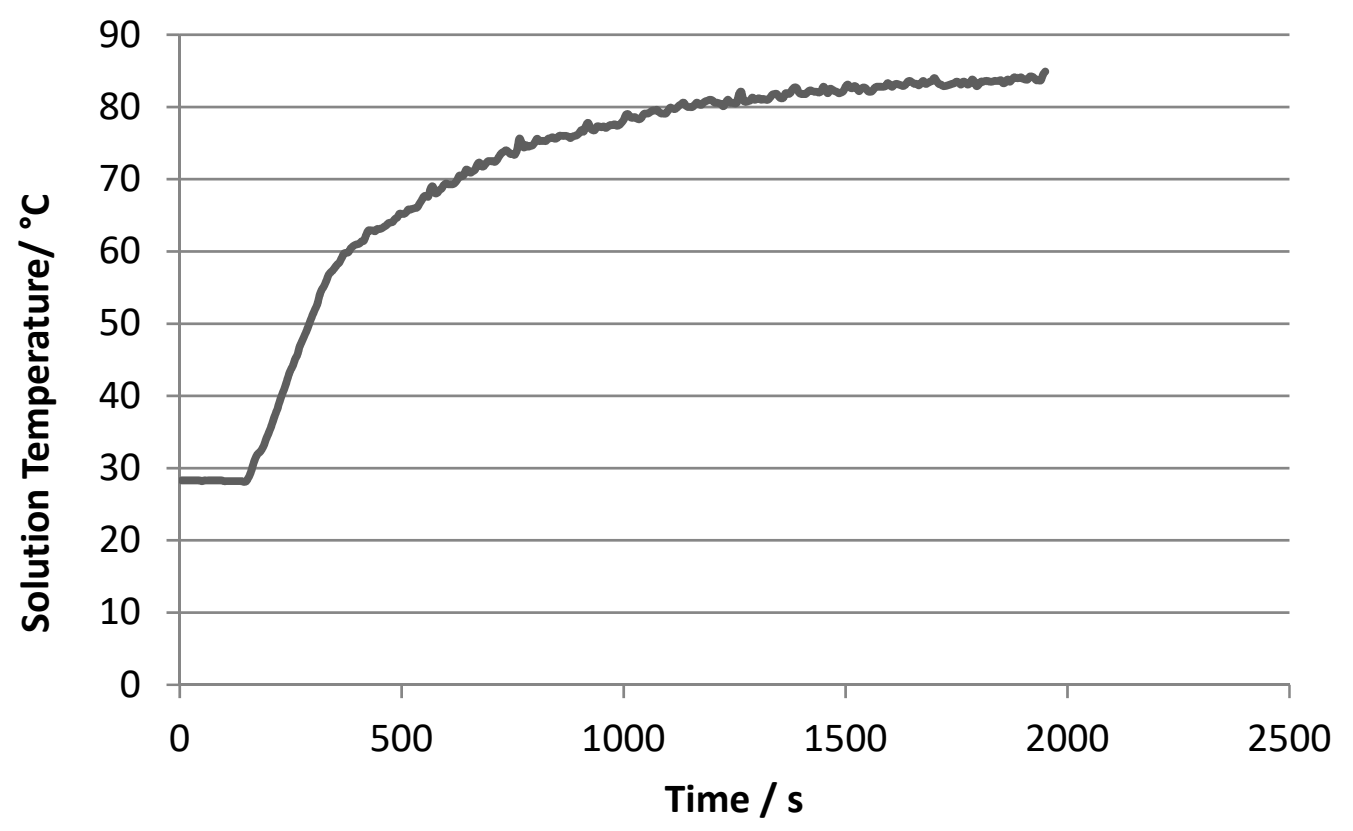

Figure 2. Variation of solution temperature in the vicinity of the sample with time, during solution plasma treatment (SPT). The temperature increased rapidly and exceeded $60^{\circ} \mathrm{C}$ within 3 min after starting SPT, and then increased slowly and reached an almost constant value of $80{ }^{\circ} \mathrm{C}$ at $30 \mathrm{~min}$.

\subsection{Characterization of Titanium Surfaces Subjected to SPT and IT in the Calcium Phosphate Solution}

\subsubsection{SEM Observation}

Figure 3 shows the SEM photographs of the polished titanium surface after SPT (Ti-SPT) (Figure 3a) and after $5 \mathrm{M} \mathrm{NaOH}$ treatment and subsequent SPT (Ti-AT-SPT) (Figure 3b). Crystals formed on both the Ti-SPT and the Ti-AT-SPT. Crystals with diameters between 5 and $20 \mu \mathrm{m}$ precipitated sparsely and 
did not cover the whole surface of the Ti-SPT, while fine spherical crystals with a diameter of $5 \mu \mathrm{m}$ precipitated and covered the whole surface of the Ti-AT-SPT.

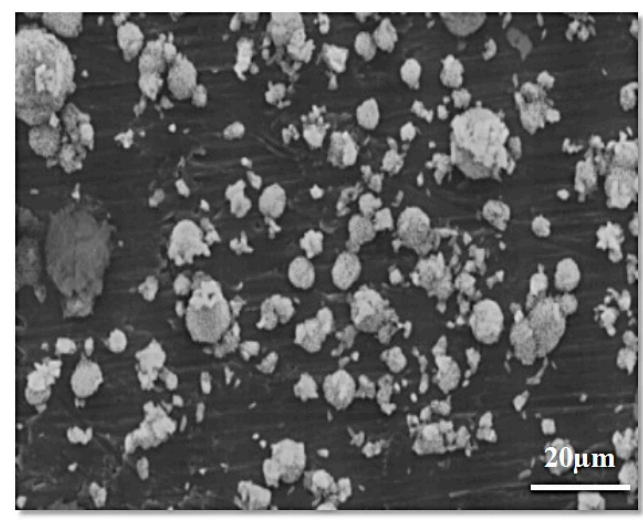

Ti-SPT

(a)

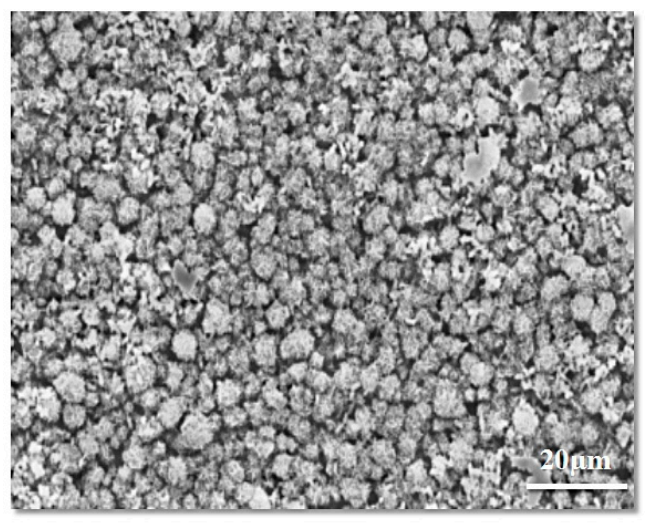

Ti-AT-SPT

(b)

Figure 3. SEM images of HA crystals precipitated on Ti-SPT (a) and Ti-AT-SPT (b). The HA spherical particles formed on both the Ti-SPT and the Ti-AT-SPT. The spherical particles precipitated sparsely and did not cover the whole surface of the Ti-SPT, while the fine spherical crystals precipitated and covered the whole surface of the Ti-AT-SPT.

Figure 4 shows the SEM photographs of the porous titanium surface after $5 \mathrm{M} \mathrm{NaOH}$ treatment and subsequent SPT (Porous-Ti-AT-SPT). Fine spherical HA particles were uniformly precipitated over the entire surface (Figure 4a) including the areas recessed in the shape of the porous structure (Figure $4 \mathrm{~b}$ ) as well as the inner surface of the pores (Figure 4c).

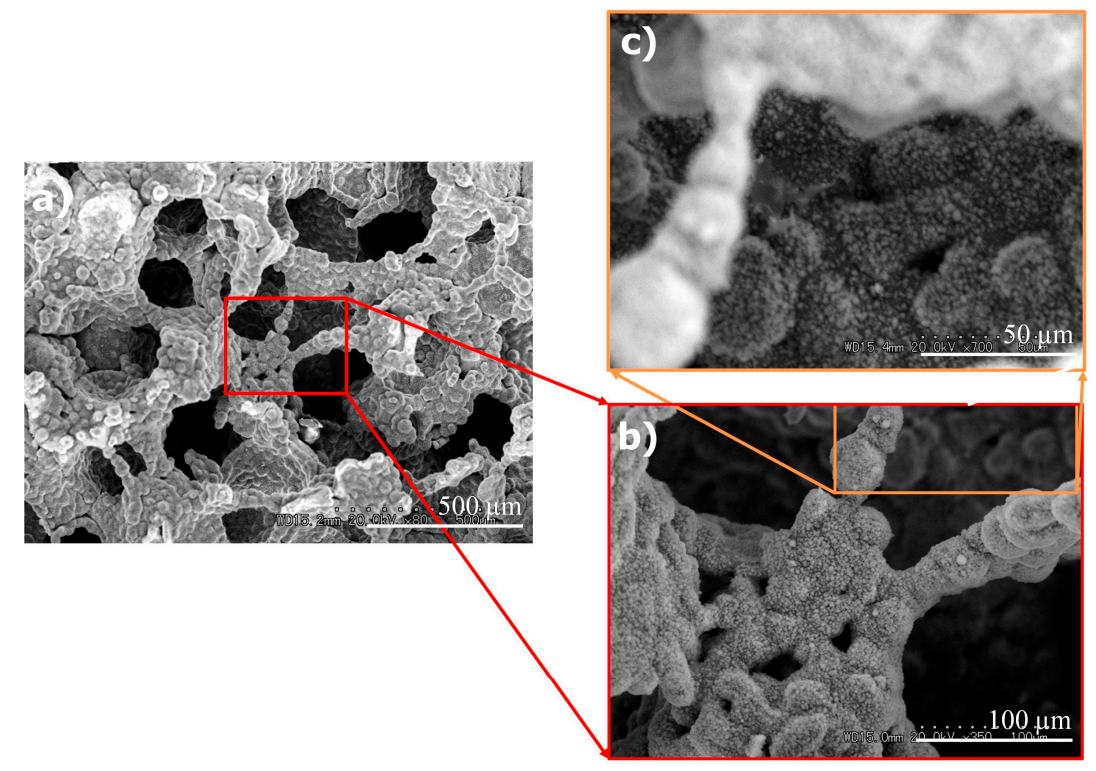

Figure 4. Scanning electron microscopy (SEM) images of Porous-Ti-AT-SPT. Fine spherical hydroxyapatite (HA) particles were uniformly precipitated over the entire surface (a), the areas recessed in the shape of the porous structure (b), and inner surface of the pores (c).

3.2.2. Analysis of Precipitated Particles on Ti-AT-SPT and Ti-AT-IT60 by XRD and EDX

Figure 5 shows the X-ray diffractogram obtained from Polished-Ti (Figure 5a), Ti-AT-SPT (Figure $5 b$ ), and synthetic HA powder (Figure $5 c$ ). The synthetic HA powder was obtained from the dried-out mineralizing solution which was subjected to SPT. 
All of the diffraction peaks were assigned to HA or the titanium substrate under the deposited film. For Ti-AT-SPT (Figure 5b), the diffraction peak of the deposited HA crystals at $25.9^{\circ}(2 \theta)$ that corresponded to the (002) lattice plane was relatively higher than the other diffraction peaks at around $32^{\circ}$, unlike that observed for the synthetic HA powder. This indicated that the spherical HA crystals that precipitated on Ti-AT-SPT were slightly oriented with the c-axis perpendicular to the titanium substrate. These results clearly demonstrated that $5 \mathrm{M} \mathrm{NaOH}$ treatment and subsequent SPT for $30 \mathrm{~min}$ in the calcium phosphate solution were effective in coating the entire surface of titanium with an HA film composed of fine crystals in a relatively short treatment time.

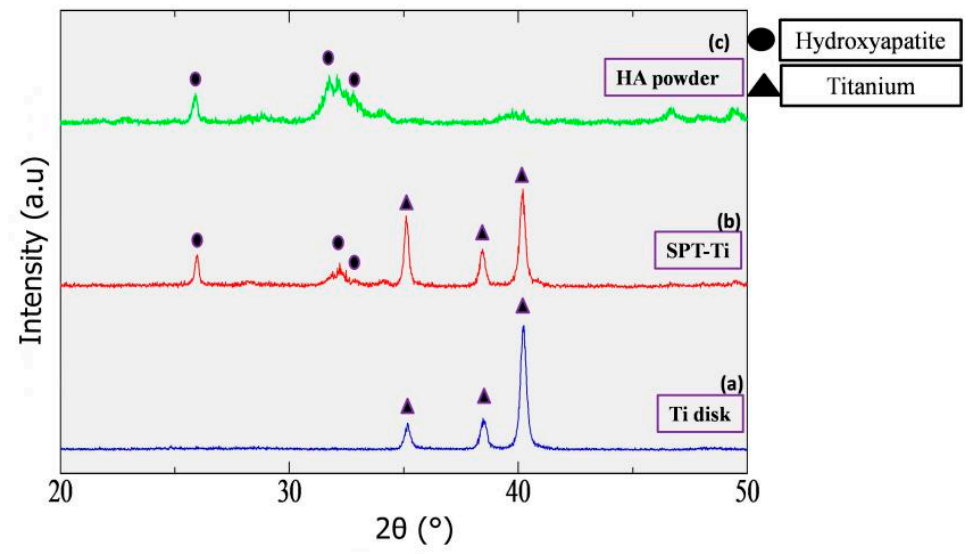

Figure 5. X-ray diffractogram obtained from (a) Polished-Ti, (b) Ti-AT-SPT, and (c) synthetic HA powder. All the diffraction peaks obtained from Ti-SPT were assigned to HA or the titanium substrate under the deposited film.

\subsection{Crystals on the Titanium Surfaces after Immersion in the Calcium Phosphate Solution}

Figure 6 shows the SEM photographs of the polished titanium after immersion in the calcium phosphate solution at $37^{\circ} \mathrm{C}\left(\mathrm{Ti}-\mathrm{IT} 37^{\circ}\right)$ and after $5 \mathrm{M} \mathrm{NaOH}$ treatment and subsequent immersion in the calcium phosphate solution at $37^{\circ} \mathrm{C}$ (Ti-AT-IT37 $7^{\circ}$. No crystals were deposited on the Ti-IT37 for up to 7 days of immersion. In contrast, spherical particles consisting of plate-like HA crystals were deposited on part of the Ti-AT-IT37 $7^{\circ}$ after 1 day of immersion. These crystals grew with time and covered almost all of the surface of the titanium after immersion for 7 days. These results were consistent with those reported previously [5].

Figure 7 shows the SEM photographs of the polished titanium after immersion in the calcium phosphate solution at $60^{\circ} \mathrm{C}\left(\mathrm{Ti}-\mathrm{IT} 60^{\circ}\right)$ and after $5 \mathrm{M} \mathrm{NaOH}$ treatment and subsequent immersion in the calcium phosphate solution at $60^{\circ} \mathrm{C}\left(\right.$ Ti-AT-IT $\left.60^{\circ}\right)$. No spherical particle precipitation was observed on the Ti-IT $60^{\circ}$ after immersion in the solution for $30 \mathrm{~min}$. Precipitated crystals between 5 and $40 \mu \mathrm{m}$ in diameter were found on the Ti-IT $60^{\circ}$ after immersion for $6 \mathrm{~h}$, and these HA spherical particles grew into large size spherical particles between 40 and $100 \mu \mathrm{m}$ in diameter after immersion for $24 \mathrm{~h}$. On the Ti-AT-IT $60^{\circ}$, small crystals precipitated after immersion for $30 \mathrm{~min}$. The density of the crystals increased due to the growth of crystals with time. From the results shown in Figures 6 and 7, it was observed that the crystal formation and its growth rate were markedly enhanced with $5 \mathrm{M} \mathrm{NaOH}$ treatment as well as an increase in the temperature of the calcium phosphate solution. 
Polished-Ti

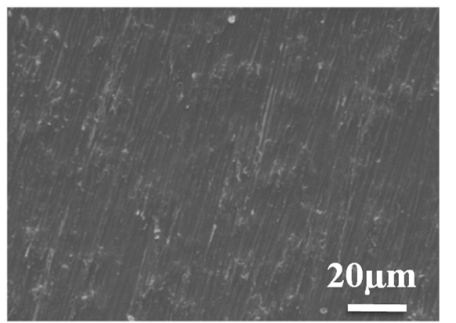

1 Days

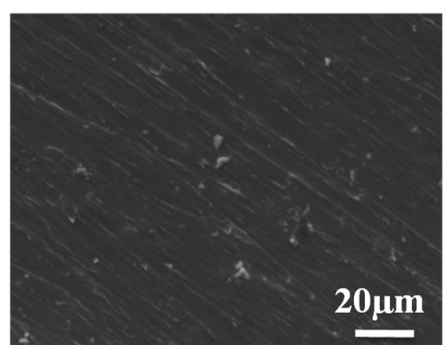

3 Days

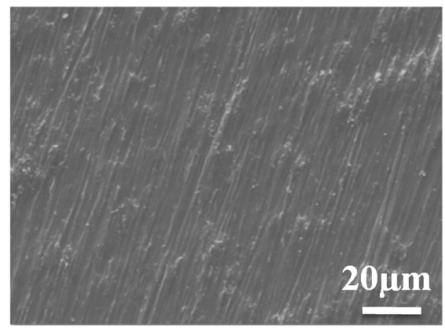

7 Days
5M NaOH-Ti

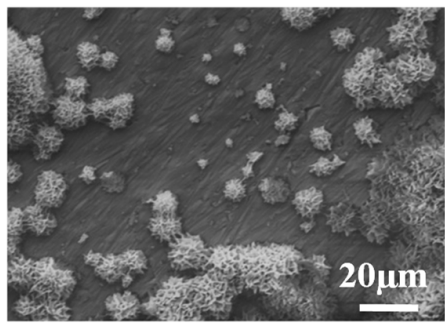

1 Days

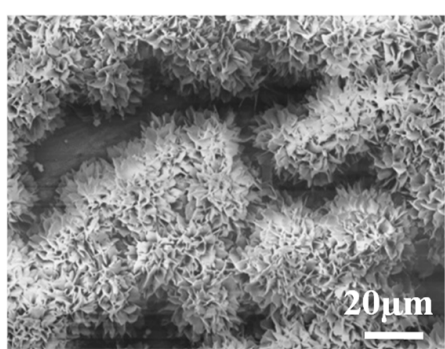

3 Days

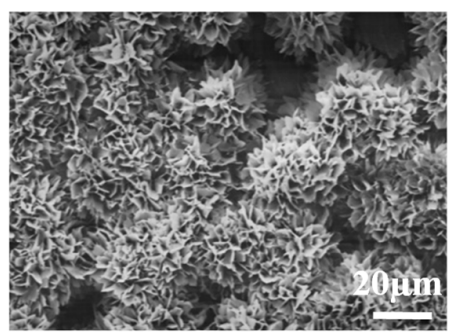

7 Days

Figure 6. SEM images of the Polished-Ti and Ti-AT after immersion in the meta stable calcium phosphate solution at $37^{\circ} \mathrm{C}\left(\mathrm{Ti}^{-\mathrm{IT}} 37^{\circ}\right.$ and Ti-AT-IT37 $7^{\circ}$. No HA spherical particles were deposited on the Ti-IT37 $7^{\circ}$ for up to 7 days of immersion. In contrast, spherical particles consisting of plate-like HA were deposited on the part of the Ti-AT-IT37 ${ }^{\circ}$ after immersion for 1 day.

Polished-Ti

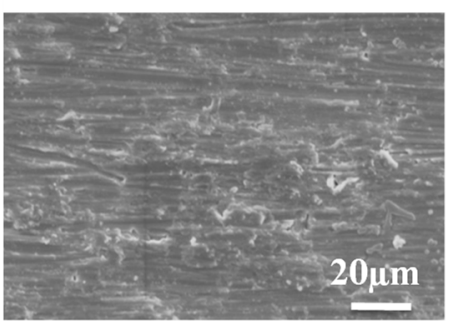

30 min

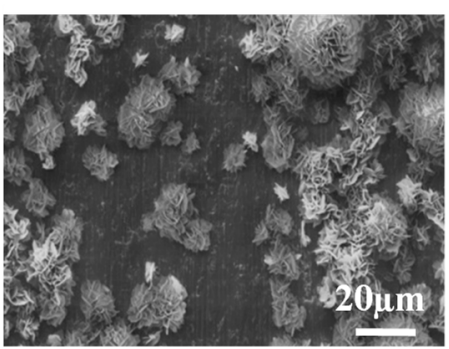

$6 \mathrm{~h}$
$5 \mathrm{M} \mathrm{NaOH}-\mathrm{Ti}$

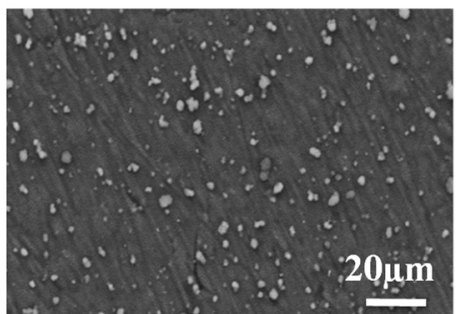

$30 \mathrm{~min}$

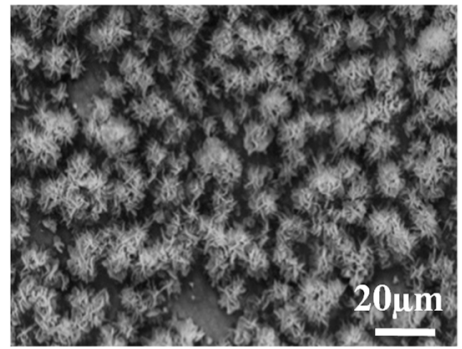

$6 \mathrm{~h}$

Figure 7. Cont. 


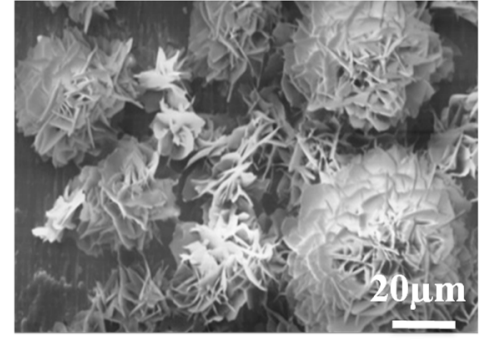

$24 \mathrm{~h}$

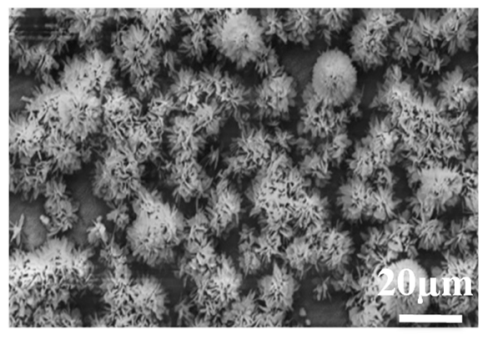

$24 \mathrm{~h}$

Figure 7. SEM images of the Polished-Ti and Ti-AT after immersion in the calcium phosphate solution at $60^{\circ} \mathrm{C}\left(\mathrm{Ti}-\mathrm{IT} 60^{\circ}\right.$ and Ti-AT-IT60 $)$. There was no HA precipitation observed on the Ti-IT60 after immersion in the solution for $30 \mathrm{~min}$. HA spherical particles were found on the Ti-IT $60^{\circ}$ after immersion for $6 \mathrm{~h}$ and they grew into large spherical particles after immersion for $24 \mathrm{~h}$. On the Ti-AT-IT60 $0^{\circ}$, small HA spherical particles precipitated after immersion for $30 \mathrm{~min}$. The spherical particles grew larger with time and covered the entire surface of a titanium after immersion for $24 \mathrm{~h}$.

\subsection{Change in the Contact Angle of Water Droplets on Polished-Ti and Ti-AT-SPT with Aging Time}

Figure 8 shows the changes in the contact angle of $20 \mu \mathrm{L}$ water droplets on Ti, Ti-AT, and Ti-AT-SPT with aging time. The contact angle value for Ti increased as the Ti disk aged, suggesting that the surface property changed from being hydrophilic to hydrophobic. This phenomenon is well known as the time-dependent degradation in biological capability or biological degradation, which arises from the absorption of hydrocarbon contaminants in air. In contrast, the contact angles for Ti-AT and Ti-AT-SPT were much lower than that for Ti throughout the aging period. The initial super hydrophilic surface remained almost unchanged up to 30 days of aging in air, although the tough hydrophilicity significantly decreased.

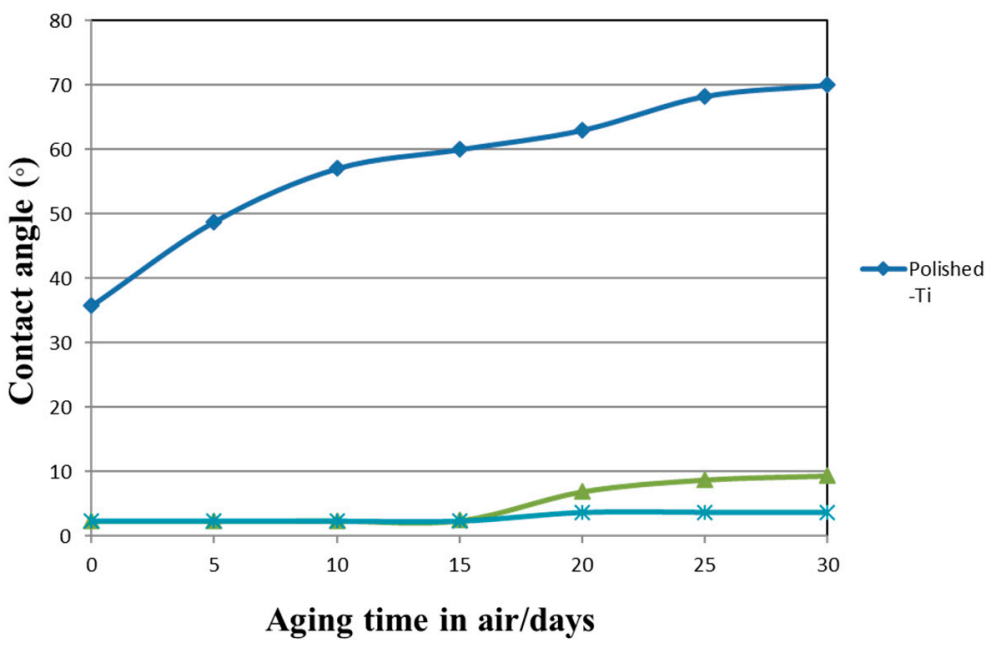

Figure 8. The changes in contact angle of water droplets on Polished-Ti, Ti-AT, and Ti-AT-SPT with aging time are shown. The contact angle for Ti-AT and Ti-AT-SPT was much lower than that for Ti throughout the aging period.

\subsection{Bioactivity Analysis}

Figure 9 shows the number of cells attached to the Polished-Ti and Ti-AT-SPT, which were subjected to aging in air for 0,7 , and 30 days, as measured with the hemocytometer. The number of MC3T3E1 cells attached to Ti-AT-SPT was approximately 50\% more than the amount attached to Ti at each aging period, which suggested that the HA coating by solution plasma treatment significantly improved the initial cell attachment $(p<0.05)$. The number of cells that attached to the Polished-Ti with 
30 days of aging in air was approximately $50 \%$ of that for the Polished-Ti without aging. In contrast, the number of attached cells on the Ti-AT-SPT after aging for 30 days remained above $85 \%$ of that for the SPT-Ti without aging.

Figure 10 shows the SEM photographs of the cells attached to Porous-Ti-AT-SPT after incubation for $4 \mathrm{~h}$. The attached cells indicated by the arrows were located not only on the outer surface (Figure 10a) but also on the surface of the concavity (Figure 10b), which indicated that the induction of bone tissue on the entire porous surface was going to occur successfully and that bone tissue growth could be expected.

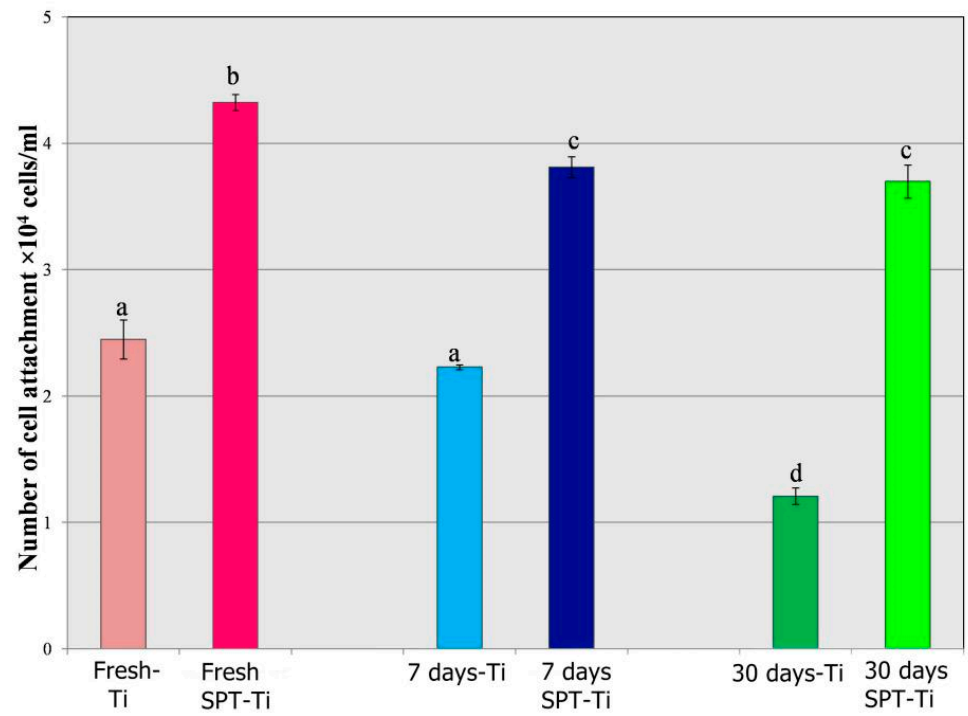

Figure 9. The number of attached cells on surfaces after $4 \mathrm{~h}$ incubation. The number of MC3T3E1 cells attached to the Polished-Ti and Ti-AT-SPT with 0,7 , and 30 days aging in air is shown in the SEM and graph. Different letters in the graph mean statistical difference at $p<0.05$.
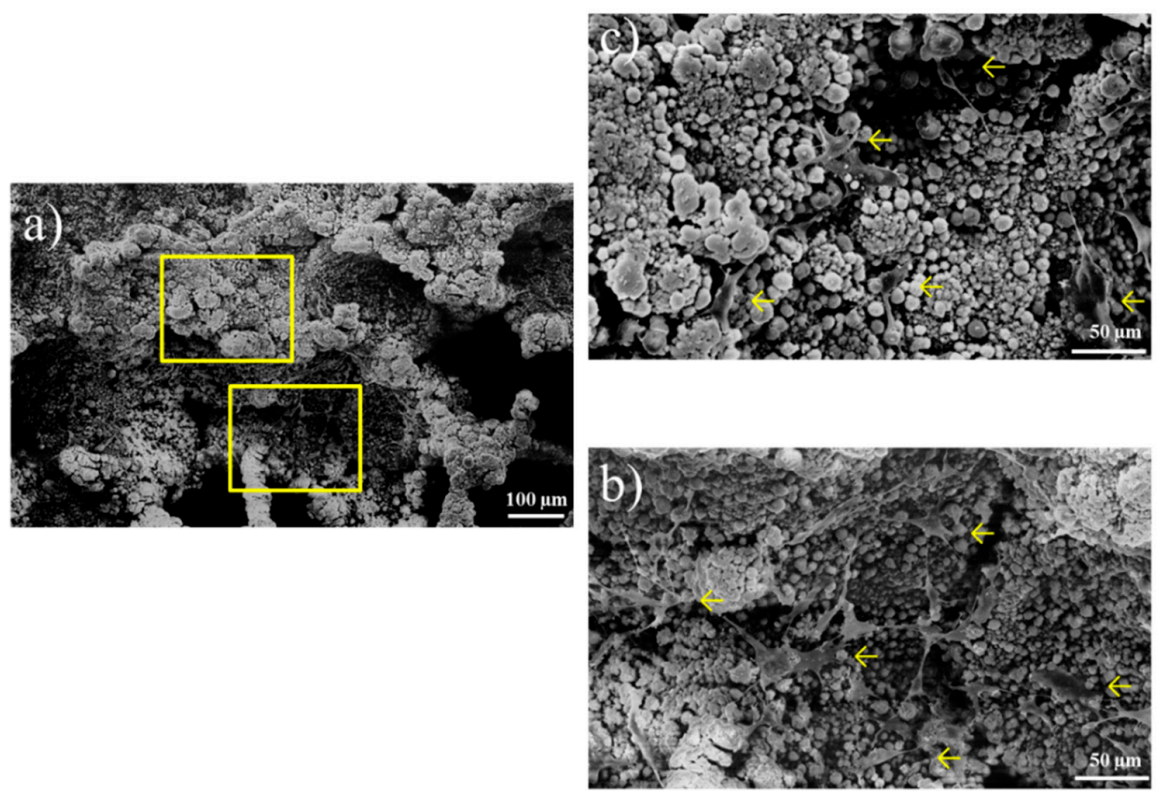

Figure 10. (a) Cell attached to porous Ti-AT-SPT after $4 \mathrm{~h}$ incubation. (b) Cell attached at the outer surface of concavity in the porous structure. (c) Cell attached at the inner surface of concavity in the porous structure. SEM images of the cells attached to Porous Ti-AT-SPT after $4 \mathrm{~h}$ incubation. The attached cells indicated by arrows were located not only on the outer surface (upper yellow square represents c) but also on the surface of concavity (lower square represents b). 


\section{Discussion}

\subsection{Process of Rapid Formation of Thin and Uniform HA Film Consisting of Fine Spherical Particles on} Ti-AT-SPT

With SPT in the calcium phosphate solution, HA spherical particles were precipitated on the Polished-Ti surface, but the size of the spherical particles was 5-20 $\mu \mathrm{m}$ in diameter. The density of the HA crystals was too low to form an HA film (Figure 3a). However, it was demonstrated that a thin and uniform HA film consisting of fine spherical HA particles with a diameter of $5 \mu \mathrm{m}$ formed on Ti-AT-SPT (Figure 3b). This rapid formation of the uniform HA film was attributed to the synergistic effect obtained by combining an alkaline treatment with SPT in a calcium phosphate solution.

The driving force for the formation of the HA film from a supersaturated solution was the change in Gibbs free energy, $\Delta G$, for transfer from a supersaturated solution to an equilibrium solution with HA crystals:

$$
\Delta G=-R T \ln S
$$

where $R$ is the gas constant; $T$ is the absolute temperature; and $S$ is the degree of supersaturation, which is expressed as:

$$
S=I P / K_{\mathrm{sp}}
$$

where $I P$ is the ionic activity and $K_{\mathrm{sp}}$ is the thermodynamic solubility product. Since the solubility product of HA, $K_{\mathrm{sp}}$, decreases with increasing temperature, it is apparent that an increase in the degree of supersaturation, $S$, together with a decrease in the $K_{\mathrm{sp}}$ value as a result of SPT was responsible for the precipitation of HA crystals on Polished-Ti. SPT for $30 \mathrm{~min}$ without alkaline treatment, however, was insufficient for the formation of a dense and uniform HA film. This probably arose from the fact that the SPT was not sufficient to lower the activation energy to enhance the formation of HA crystals (Figure 11a) and a few critical and supercritical HA nuclei were induced by SPT, as shown in Figure 12a.

(a)

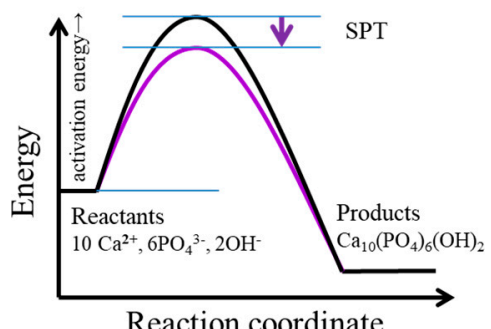

Reaction coordinate (b)

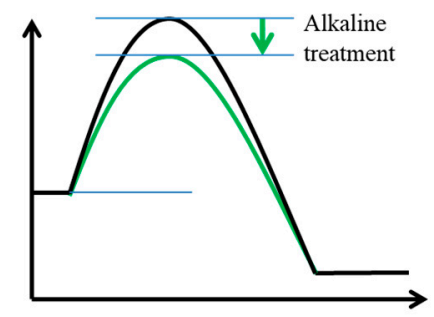

(c)

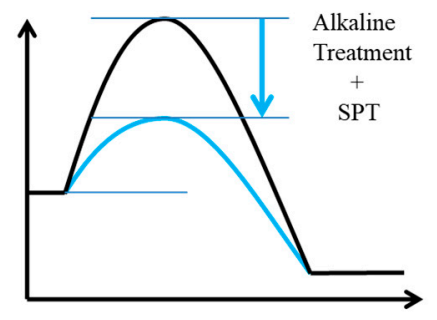

Figure 11. Energy profile in the hydroxyapatite formation. Activation energy of hydroxyapatite formation was lowered by (a) SPT and (b) alkaline treatment, and was notably lowered by (c) SPT and alkaline treatment and SPT. 
(a) SPT

Sparse nucleation

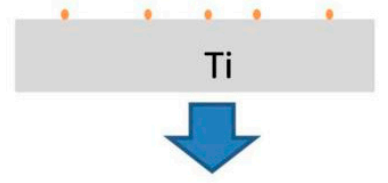

Irregular precipitation of HA (b) Alkaline treatment

Sparse nucleation

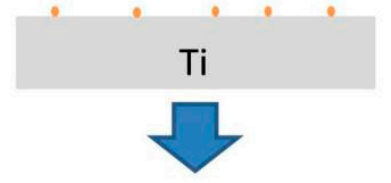

Irregular precipitation of $\mathrm{HA}$ with large and grown crystals

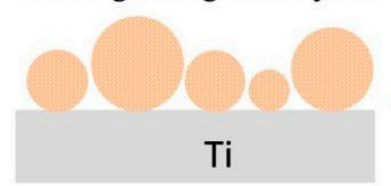

(c) Alkaline treatment + SPT

Dense nucleation

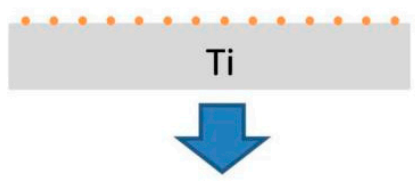

Uniform precipitation of HA with round \& fine shaped crystals

Figure 12. Schematic illustration of HA nucleation and HA crystal growth on the titanium surfaces. The appearance of particles formed on the Ti surface was quite different among (a) Ti-SPT, (b) Ti-AT-IT, and (c) Ti-AT-SPT.

With $5 \mathrm{M} \mathrm{NaOH}$ treatment, a sodium titanate hydrogel layer formed on the polished titanium [5]. It was also found that the hydrogel layer quickly released sodium ions with the uptake of calcium ions when alkaline-treated Ti was soaked in calcium phosphate solution. This ion exchange reaction was proposed to take place very quickly to maintain the electrical neutrality of the hydrogel and increased the calcium ion concentration at the hydrogel surface. Increase in $I P$, as a result of a higher calcium ion concentration, also increased the degree of supersaturation with respect to HA, which enhanced the apatite nucleation in a mineralizing solution [13]. In this case, the activation energy required for HA nucleation was also insufficiently lowered by the increased concentration of Ca ions (Figure 11b) to induce a few nuclei (Figure 12b). The HA crystals grew larger with an increase in soaking time in a calcium phosphate solution, and a thick HA film consisting of coarse HA spherical particles was formed after soaking for 1 day at $60^{\circ} \mathrm{C}$ (Figure 11b).

With the combination of alkaline treatment and SPT, the degree of supersaturation, $S$, was markedly increased with both an increase in $I P$ and a decrease in $K_{\text {sp }}$. The activation energy required for HA nucleation was probably lowered sufficiently (Figure 11c) to induce a large number of HA nuclei as shown in Figure 12c. As a result, a thin and uniform HA film consisting of fine spherical particles could be obtained (Figure 12c). As it is a uniform precipitation with fine and round HA particles, the height of the precipitation is equal to the height of HA crystal, which is $1 \mu \mathrm{m}$.

\subsection{Chemical and Biological Properties of HA Film Formed by SPT}

Previous studies have revealed that titanium undergoes a time-dependent degradation in biological capability due to the unavoidable contamination of the titanium surfaces by hydrocarbons in air [14]. Contact angle measurements of the water droplets demonstrated that the titanium surface changed from being hydrophilic to hydrophobic with an increase in aging time (Figure 8). This study demonstrated that the contact angle value for a HA-coated titanium disk (Ti-AT-SPT) was significantly lower than that for polished titanium throughout the aging periods. In addition to this, the initial super hydrophilicity of the HA-coated titanium surface by SPT was found to be maintained after 30-day aging in air (Figure 8). Unlike titanium, the HA-coated titanium did not show age-related impaired bioactivity, which is defined as biological aging [15]. The reason for the highly hydrophilic nature of HA-coated titanium can be attributed to the characteristic of HA crystals as HA is an ionic crystal with an abundance of ionic sites that attract water molecules.

The number of MC3T3E1 cells attached to Ti-AT-SPT was approximately $50 \%$ larger than that of Polished-Ti at each aging period, suggesting that the HA coating by solution plasma treatment 
significantly improved the initial cell attachment. This may be one of the reasons why HA-coated titanium implants possess better cytocompatibility and facilitate rapid bone formation due to their excellent osteoconductive property.

These results suggest that an osteoconductive porous-surfaced titanium implant can be developed with a HA coating by solution plasma treatment in a calcium phosphate solution in a short time.

\subsection{HA Film Coating on Titanium by SPT to Develop an Osteoconductive Porous-Surfaced Dental Implant Body}

A HA film coating was achieved when Ti-AT was soaked in calcium phosphate solution at $37^{\circ} \mathrm{C}$ for seven days and at $60^{\circ} \mathrm{C}$ for one day (Figures 6 and 7).

In a $37^{\circ} \mathrm{C}$ immersion treatment, a HA film coating was achieved when polished-Ti and Ti-AT were soaked in calcium phosphate solution for seven days. The approximate size of the precipitated HA spherical particles on Ti-AT was $40-50 \mu \mathrm{m}$. In a $60^{\circ} \mathrm{C}$ immersion treatment, an HA film coating was achieved when the Polished-Ti and Ti-AT were soaked in the calcium phosphate solution for $24 \mathrm{~h}$. The approximate size of the precipitated HA spherical particles on Polished-Ti was more than $40 \mu \mathrm{m}$, and was $20 \mu \mathrm{m}$ on Ti-AT.

These results suggest that the size of the HA spherical particles precipitated during the immersion treatments in the calcium phosphate solution was too large to coat the titanium implant with a porous surface structure. Furthermore, in the $37^{\circ} \mathrm{C}$ immersion, there was less precipitation compared to the $60^{\circ} \mathrm{C}$ immersion. For a dense precipitation of HA, the temperature must be $60^{\circ} \mathrm{C}$ or more. Temperature of the mineralizing solution must be $37^{\circ} \mathrm{C}$ during the initializing of the solution plasma treatment.

With $5 \mathrm{M} \mathrm{NaOH}$ treatment and subsequent SPT in the calcium phosphate solution, dense nucleation with critical size nuclei was successfully precipitated on the sample (Figure 12c) and a thin and dense HA layer consisting of fine spherical crystals was formed on the titanium disk surface after SPT for 30 min (Figure 3b).

The solution plasma surface modification for coating HA onto titanium proceeds in a wet process unlike other HA surface methods using a dry process. Therefore, the coating of a HA film consisting of fine crystals on the entire surface of titanium implant bodies with porous surface structures can be achieved. MC3T3E1 cells were observed not only on the outer surface, but also on the surface of the concavity (Figure 10), indicating that the induction of bone tissue on the entire porous surface would occur successfully and that bone tissue ingrowth could be expected. To enhance the growth of mineralized tissue into the pore spaces and to keep the vascular system interconnected by the pores for continued bone development, the porous structure of a titanium implant provides a unique biological bone anchorage to the titanium implants. Enhancement of bone/implant mechanical bonding can allow dentists to use dental implant bodies with a short length, which can greatly contribute to an increase in the ability of dental implants to be used for a wide variety of treatment options, especially for patients with low bone quality and with advanced maxillary/mandibular residual ridge resorption.

\subsection{Features of the SPT for HA Film Coating}

A HA film coating on titanium was achieved by combining a simple chemical treatment (5 M $\mathrm{NaOH}$ treatment) with SPT in a calcium phosphate solution. One of the striking features of this proposed method was that the rate of HA film formation during SPT was faster than that in most wet processes. For example, Kim et al. reported that a bone-like apatite layer formed on the $5 \mathrm{M} \mathrm{NaOH}$ treated titanium substrate when the substrate had been soaked in simulated body fluid (SBF) for more than one day [5]. In contrast, a uniform HA film was observed to form on the $5 \mathrm{M} \mathrm{NaOH}$ treated titanium by SPT for only $30 \mathrm{~min}$ (Figure 3b). As stated before, the thermal energy produced during SPT increased the degree of solution supersaturation with respect to HA, and thus greatly increased the growth rate of the HA crystals. 
The substrate heating method in liquid with the application of a large current can be applied to electrically conducting materials such as metals and alloys. For SPT, the solution in contact with titanium is heated with the thermal energy generated by plasma, suggesting that this HA coating method can be applied to materials without electrical conductivity such as ceramics.

\section{Conclusions}

The present study demonstrated that it is possible to acquire a homogenous precipitation of hydroxyapatite over the entire surface of a smooth and porous titanium disk by the implementation of a solution plasma surface modification treatment in a mineralizing solution. The solution plasma-treated samples showed significantly better cyto-compatibility than the other specimens.

Author Contributions: Conceptualization K.E.; Methodology, K.E. and T.S.; Software, A.B.D. and M.R.H.; Validation, F.N.-T. and T.N.; Formal Analysis, A.B.D., T.N. and F.N.-T.; Investigation, A.B.D.; Resources, K.E.; Data Curation, A.B.D.; Writing-Original Draft Preparation, K.E. and A.B.D.; Writing-Review \& Editing, T.S. and M.R.H.; Visualization, A.B.D. and M.R.H.; Supervision, K.E. and T.N.

Funding: This research received no external funding.

Acknowledgments: The authors would like to acknowledge the Division of Orthodontics and Dentofacial Orthopedics, School of Dentistry, Health Sciences University of Hokkaido for their technical support.

Conflicts of Interest: The authors declare no conflict of interest.

\section{References}

1. Dental Implants Facts and Figures. Available online: https://www.aaid.com/about/Press_Room/Dental_ Implants_FAQ.html (accessed on 1 December 2018).

2. Herman, H. Plasma spray deposition processes. MRS Bull. 1988, 12, 60-67. [CrossRef]

3. Dasarathy, H.; Riley, C.; Coble, H.D. Analysis of apatite deposits on substrates. J. Biomed. Mater. Res. 1993, 27, 477-482. [CrossRef] [PubMed]

4. Ong, J.L.; Harris, L.A.; Lucas, L.C.; Lacefield, W.R.; Rigney, D. X-ray photoelectron spectroscopy characterization of ion-beam sputter-deposited calcium phosphate coatings. J. Am. Ceram. Soc. 1991, 74, 2301-2304. [CrossRef]

5. Kim, H.M.; Miyaji, F.; Kokubo, T.; Nishiguchi, S.; Nakamura, T. Graded surface structure of bioactive titanium prepared by chemical treatment. J. Biomed. Mater. Res. 1999, 45, 100-107. [CrossRef]

6. Ban, S.; Maruno, S.; Harada, A.; Hattori, M.; Narita, K.; Hasegawa, J. Effect of temperature on morphology of electrochemically deposit calcium phosphates. Dent. Mater. J. 1996, 15, 31-38. [CrossRef] [PubMed]

7. Ishikawa, K.; Miyamoto, Y.; Nagayoma, M.; Asaoka, K. Blast coating method: New method of coating titanium surface with hydroxyapatite at room temperature. J. Biomed. Mater. Res. 1997, 38, 129-134. [CrossRef]

8. Kuroda, K.; Ichino, R.; Okido, M.; Takai, O. Hydroxyapatite coation on titanium by thermal substrate method in aqueous solution. J. Biomed. Mater. Res. 2001, 59, 390-397. [CrossRef] [PubMed]

9. Tamura, M.; Endo, K.; Maida, T.; Ohno, H. HA film coating by the thermally induced liquid-phase deposition method for titanium implants. Dent. Mater. J. 2006, 25, 32-38. [CrossRef] [PubMed]

10. Saito, G.; Nakasugi, Y.; Akiyama, T. Generation of solution plasma over a large electrode surface area. J. Appl. Phys. 2015, 118, 23303. [CrossRef]

11. Kokubo, T.; Kim, H.M.; Kawashita, M.; Nakamura, T. Bioactive metals: Preparation and properties. J. Mater. Sci. Mater. Med. 2004, 15, 99-107. [CrossRef] [PubMed]

12. Saito, T.; Arsenault, A.L.; Yamauchi, M.; Kuboki, Y.; Crenshaw, M.A. Mineral Induction by immobilized phosphoproteins. Bone 1997, 21, 305-311. [CrossRef]

13. Nygren, H. Initial reactions of whole blood with hydrophilic and hydrophobictitanium surface. Colloids Surf. B Biointerfaces 1996, 6, 329-333. [CrossRef] 
14. Jia, S.; Zhang, Y.; Ma, T.; Chen, H.; Lin, Y. Enhanced hydrophilicity and protein adsorption of titanium surface by sodium bicarbonate solution. J. Nanomater. 2015, 2015, 5. [CrossRef]

15. Att, W.; Hori, N.; Takeuchi, M.; Ouyang, J.; Yang, Y.; Anpo, M.; Ogawa, T. Time-dependent degradation of titanium osteoconductivity: An implication of biological aging of implant materials. Biomaterials 2009, 30, 5352-5363. [CrossRef] [PubMed] 